\title{
Le don de cellules souches du sang
}

\author{
Jörg Haltera, Simone Romagnolib \\ a Hématologue, MD, Hôpital universitaire de Bâle \\ b Assistant scientifique, PhD, Projet FNS, Université de Bâle
}

La transplantation de cellules souches du sang (hematopoüetic stem cells transplantation, HSCT) est une thérapie reconnue pour soigner des maladies malignes du sang (leucémies, lymphomes, myélomes, etc.) et des dysfonctionnements acquis ou héréditaires (tels que des anémies ou des déficiences immunitaires). L'approche consiste principalement à substituer le système hématopoïétique défaillant du patient avec un nouveau système développé à partir des cellules souches transplantées, prélevées chez un donneur le plus souvent non apparenté. Il s'agit donc d'une thérapie pionnière dans le domaine de la médecine régénérative et elle représente un modèle pour les thérapies futures utilisant des cellules souches.

La HSCT exige une collaboration internationale. En août 2012, presque 20 millions de personnes et 500000 unités de sang du cordon étaient inscrites dans l'un des 67 registres de donneurs ou 45 banques publiques de sang du cordon à travers le monde. Il arrive ainsi fréquemment que le donneur et le receveur appariés habitent dans des pays ou des continents différents. Cela implique une logistique et une organisation méticuleuses. Cependant, malgré ces chiffres impressionnants et les efforts consentis, il n'est pas toujours possible de trouver un phénotype HLA compatible pour un patient donné. Pour cette raison, il est important de poursuivre ces efforts dans plusieurs directions, à savoir le recrutement des donneurs (y compris d'origine non caucasienne, groupe ethnique le mieux représenté dans les registres de donneurs), l'innovation diagnostique et thérapeutique, la promotion de standards élevés de bonnes pratiques et la réalisation de recherches liées à l'utilisation de cellules souches du sang.

Tous ces objectifs soulèvent des questions normatives complexes, qui vont de la protection des données personnelles, de l'adaptation des régimes de consen- tement pour des projets de recherche impliquant des analyses génétiques nouvelles, à l'utilisation ou à la réutilisation d'échantillons de matériel biologique conservés dans des biobanques, aux stratégies innovantes de recrutement de donneurs, à la sauvegarde de l'autonomie, de l'intégrité et du bien-être des personnes impliquées. Pour ces raisons le projet «Mastering the legal and ethical challenges of present and future cell donation: Risk assessment and contingency to ensure donor protection as well as competitiveness of regenerative medicine in Switzerland» financé par le Fonds National Suisse dans le cadre du Programme National de Recherche NRP63 Stem Cells and Regenerative Medicine s'est donné pour objectif d'étudier quelques-uns des enjeux juridiques et éthiques majeurs liés à cette thérapie. Parmi ses activités, le projet a organisé en mars 2012 un colloque réunissant des experts internationaux, dont sont issues quelques-unes des contributions présentées ici. Le présent numéro de Bioethica Forum se veut une fenêtre ouverte sur un domaine moins connu de la médecine de transplantation. Un domaine traversé par des enjeux éthiques et juridiques stimulants. En effet, de par sa nature propre, la HSCT évolue continuellement - à la limite - entre la clinique et la recherche génétique, entre les Etats et les continents, entre des systèmes juridiques et des normes éthiques, entre des sensibilités culturelles et politiques. C'est pourquoi la HSCT mérite une place dans le débat bioéthique contemporain.

\section{Correspondance}

Dr Jörg Halter

Universitätsspital Basel

Abteilung Hämatologie

Spitalstrasse 21/Petersgraben 4

$\mathrm{CH}-4031$ Basel

E-mail: jhalter[at]hin.ch

simofilo[at]gmail.com 